\title{
Neuer Antikörper birgt Potenzial zum Wandel der Behandlung einer seltenen Form von Psoriasis
}

Das The New England Journal of Medicine (NEJM) hat kürzlich neue Daten einer klinischen Phase-I-Studie veröffentlicht, die zeigen, dass BI 655130, eine experimentelle „First-in-class“-Behandlung, die Symptome einer Generalisierten Pustulösen Psoriasis (GPP) signifikant lindert. BI 655130 ist ein monoklonaler Antikörper, der die Wirkung des Interleukin-36-Rezeptors (IL 36R) blockiert.

Die klinischen Daten, die auch auf der letzten Jahrestagung der American Academy of Dermatology (AAD) in Washington D.C. vorgestellt wurden [1], deuten darauf hin, dass BI 655130' bei sieben GPP-Patienten mit akuten moderaten oder schwerwiegenden Entzündungsschüben für eine schnelle Linderung der Symptome sorgte, die bis zum Studienende (Woche 20) aufrecht-erhalten wurde [2].

„Das genaue Abzielen auf den IL-36-Signalweg ist eines der aufregendsten Gebiete der dermatologischen Forschung. Fortschritte in Bezug auf diesen Mechanismus wurden von der Wissenschaftsgemeinschaft sehnlichst erwartet", so der verantwortliche Prüfer, Professor Hervé Bachelez vom Hôpital Saint- Louis in Paris, Frankreich. „Diese Studie liefert seit Langem erhoffte Daten, die den positiven Effekt des Blockierens der Wirkung von IL 36 als einen neuen Behandlungsansatz belegen. Die beobachtete schnelle Verbesserung bei den Patienten nach einer einzelnen Dosis BI 655130 deutet auf großes Potenzial für die künftige Behandlung von GPP hin."

Die seltene GPP ist eine chronische Erkrankung, die sich von der weiter verbreiteten Plaque-Psoriasis unterscheidet [3]. Die Haut wird rot und

Bl 655130 ist ein Prüfpräparat. Sicherheit und Wirksamkeit wurden noch nicht nachgewiesen. es kommt zu einem großflächigen Ausschlag mit zahlreichen Bläschen und nicht-infektiösem Eiter [4-6]. Außerdem kann es bei GPP zum plötzlichen Auftreten von Fieber, Schüttelfrost und schmerzhaften Hautläsionen kommen [4-6] GPP kann mit lebensbedrohlichem Organversagen und infektiösen Komplikationen einhergehen und sollte deshalb als medizinischer Notfall betrachtet werden [7]. Die verfügbaren Behandlungsoptionen sind stark begrenzt, und es mangelt ihnen an tiefgehender und langanhaltender Wirksamkeit. Aus diesem Grund gibt es bei GPP einen großen Bedarf nach neuen Behandlungsoptionen mit schneller, starker und langanhaltender Wirksamkeit [8].

„BI 655130 ist ein neuer, von Boehringer Ingelheim entdeckter Antikörper, der zur Behandlung mehrerer Entzündungskrankheiten mit der Hoffnung untersucht wird, die derzeit verfügbaren Therapien für die betroffenen Patienten zu wandeln", so Dr. Jan Poth, Therapiebereichsleiter CNS und Immunologie bei Boehringer Ingelheim.

\section{Literatur}

1. Krueger JG, Bachelez H, Choon SE, et al. The therapeutic efficacy of BI 655130, an antiinterleukin-36 receptor antibody, in patients with acute generalized pustular psoriasis is associated with the downregulation of inflammatory and tissue remodeling genes in lesional skin. Oral presentation, annual meeting of the American Academy of Dermatology, March 1-5 2019, Washington D.C.

2. Bachelez H, Choon SE, Marrakchi S et al (2019) Inhibition of the IL-36 Pathway for the Treatment of Generalized Pustular Psoriasis. N Eng J Med 380(10):981-983

3. Navarini AA, Burden AD, Capon F et al (2017) European consensus statement on phenotypes of pustular psoriasis. J Eur Acad Dermatol Venereol 31:1792-1799

4. Umezawa Y, Ozawa A, Kawasima T et al (2003) Therapeutic guidelines for the treatment of generalized pustular psoriasis (GPP) based on a proposed classification of disease severity. Arch Dermatol Res 295(Suppl 1):S43-54

5. Viguier M, Allez M, Zagdanski AM et al (2004) High frequency of cholestasis in generalized pustular psoriasis: Evidence for neutrophilic involvement of the biliary tract. Hepatology 40(2):452-458

6. Augey F, Renaudier P, Nicolas JF (2006) Generalized pustular psoriasis (Zumbusch): a French epidemiological survey. Eur J Dermatol 16(6):669-673

7. Practical Dermatology (2019) Addressing Localized and Generalized Pustular Psoriasis-A Potential Medical Emergency. http://practicaldermatology.com/2010/11/ addressing-localized-and-generalizedpustular-psoriasisa-potential-medicalemergency (Erstellt: 02.2019)

8. Robinson A, Van Voorhees AS, Hsu S et al (2012) Treatment of pustular psoriasis: from the Medical Board of the National Psoriasis Foundation. J Am Acad Dermatol 67(2):279-288

hautnah $2019 \cdot 18: 53$

https://doi.org/10.1007/s12326-0190335-7

(c) Springer-Verlag GmbH Austria, ein Teil von Springer Nature 2019
Quelle: Presseaussendung der Boehringer Ingelheim RCV GmbH \& Co KG 